\title{
The analysis of the method on the one variable function's limit
}

\author{
Ke Wu
}

Department of Mathematics and Statistics, Zaozhuang University, Zaozhuang 277160, China

Keywords: Function of one variable; the limit; Evaluation method.

\begin{abstract}
The limit of one variable function is an important problem in Higher Mathematics, it is a basic method to study the relationship between the function of variables. This template describes some important method about counting the limit, and gives the solving skills.
\end{abstract}

\section{Introduction}

The concept of limit is produced in order to solve some practical problems of precise. In the third Century A.D. China's well-known mathematician Liu Hui used the method of cyclostome.

Surgery, and used the regular polygon inscribed in a circle to approximated the area of a circle, this is the estimating thinking. Liu hui starts from the hexagon inscribed in a circle, does the Inscribed regular twelve sided in shape, twenty-four polygon etc., doubles the number of edges every time. So we get a series of inscribed regular polygon areas $A_{1} A_{2} A_{3} \ldots, A_{n} \ldots$. Imagine $n$ infinite increase, and the number of edges inscribes regular infinite increase. In this process, inscribed regular infinite closes to a circle. At the same time, $A_{n}$ inhibiting closes to a determined value, the determined values can be understood as the area of a circle. This is the origin of the conception of limit.

The limit of one variable function is an important problem in Higher Mathematics. Limit method is a basic method to study the relationship between the function of variables, it is an important basis for the limit of function of many variables, the derivative, differential and integral calculus.

The limit is an important foundation of calculus, it is necessary to make a summary and analysis for the method of solving the limit, prepare for Calculus Learning.

\section{The various method of finding the limit of various}

\section{Calculation of limit by using using the concept}

Definition 1 assuming the function $f(x)$ at the point $x_{0}$ of a deleted neighborhood has a definition. If there exists a constant $A$, for any positive number, there is always a positive number, when $0<\left|x-x_{0}\right|<\delta$, there have been $|f(x)-A|<\varepsilon$, then the constant $A$ is called the limit function when $x \rightarrow x_{0}$, write as:

$$
\lim _{x \rightarrow x_{0}} f(x)=A .
$$

Example 1 Verification: when $t_{0}>0 \lim _{t \rightarrow t_{0}} \sqrt{t}=\sqrt{t_{0}}$.

Prove $\forall \varepsilon>0$ as:

In order to make

$$
\left|\sqrt{t}-\sqrt{t_{0}}\right|=\left|\frac{t-t_{0}}{\sqrt{t}+\sqrt{t_{0}}}\right| \leq \frac{1}{\sqrt{t_{0}}}\left|t-t_{0}\right|,
$$

$$
\left|\sqrt{t}-\sqrt{t_{0}}\right|<\varepsilon,
$$

As long as

$$
\left|t-t_{0}\right|<\sqrt{t_{0}} \varepsilon
$$

So make 


$$
\delta=\sqrt{t_{0}} \varepsilon
$$

When

$$
0<\left|t-t_{0}\right|<\delta,
$$

The value of corresponding functions $\sqrt{t}$ meet

$$
\left|\sqrt{t}-\sqrt{t_{0}}\right|<\varepsilon .
$$

According to the definition of limit of function:

$$
\lim _{t \rightarrow t_{0}} \sqrt{t}=\sqrt{t_{0}} .
$$

Calculation of limit by definition is the most directed method, the key is to determine suitable

Positive values $\delta$, we can use the scaling method to identify the positive value. In addition, using the definition of limit is directed but it is not simple method, we need other methods to limit the auxiliary.

\section{Calculation of limit by using properties of infinitesimal}

Theorem 1 A product of bounded function and an infinitesimal is an infinitesimal.

Example 2 Counting $\lim _{t \rightarrow \infty} \frac{\sin t}{t}$.

Analysis: $\frac{\sin t}{t}$ is the product an $\sin t \operatorname{ad} \frac{1}{t}$. Because $\frac{1}{t}$ is the infinitesimal when $t \rightarrow \infty$ and $\sin t$ is bounded function. According Theorem 1,

$$
\lim _{t \rightarrow \infty} \frac{\sin t}{t}=0 .
$$

When using Theorem 1, be sure to see the change process of variables, judge clearly which is

Bounded function, which is the infinitesimal, Only the Multiplication about a bounded function and an infinitesimal is an infinitesimal multiplication. As in example 2, if $t \rightarrow 0$, then $\frac{1}{t}$ is not infinitesimal, but infinite. In fact, we know that:

\section{Calculation of limit by using squeezing rule}

$$
\lim _{t \rightarrow 0} \frac{\sin t}{t}=1
$$

Theorem 2 If when

$$
t \in \stackrel{0}{U}\left(t_{0}, r\right), g(t) \leq f(t) \leq h(t)
$$

And

$$
\lim _{t \rightarrow t_{0}} g(t)=A \lim _{t \rightarrow t_{0}} h(t)=A
$$

Then

Example 3 Counting $\lim _{t \rightarrow 0^{+}} t\left[\frac{1}{t}\right]$

$$
\lim _{t \rightarrow t_{0}} f(t)=A .
$$

Count: As

$$
\frac{1}{t}-1 \leq\left[\frac{1}{t}\right] \leq \frac{1}{t},
$$

When $t \in(0,1)$ because $\lim _{t \rightarrow 0^{+}}\left(\frac{1}{t}-1\right) t=1$ And $\lim _{t \rightarrow 0^{+}} t \frac{1}{t}=1$, then $\lim _{t \rightarrow 0^{+}} t\left[\frac{1}{t}\right]=1$.

Analysis: Calculation of limit by using squeezing rule, the key is to determine $g(t) h(t)$, and $\lim _{t \rightarrow t_{0}} g(t)=\lim _{t \rightarrow t_{0}} h(t)=\mathrm{A}$.

\section{Calculation of limit by the equivalent infinitesimal}

Theorem 3 When:

$$
\partial \square \partial^{\prime}, \beta \square \beta^{\prime},
$$


And $\lim \frac{\beta^{\prime}}{\alpha^{\prime}}$ exist, so

$$
\lim \frac{\beta}{\alpha}=\lim \frac{\beta^{\prime}}{\alpha^{\prime}}=\lim \frac{\beta}{\alpha^{\prime}}=\lim \frac{\beta^{\prime}}{\alpha} .
$$

The commonly used equivalent infinitesimal: When $t \rightarrow 0$,

$$
\sin t \square t, \tan t \square t, \arcsin t \square t, \arctan t \square t, 1-\cos t \square \frac{1}{2} t^{2},(1+t)^{\frac{1}{n}}-1 \square \frac{1}{n} t .
$$

Example 4 Counting $\lim _{t \rightarrow 0} \frac{\left(1+t^{2}\right)^{\frac{1}{3}}-1}{\cos t-1}$

Count: When $t \rightarrow 0,\left(1+t^{2}\right)^{\frac{1}{3}}-1 \square \frac{1}{3} t^{2} \cos t-1 \square-\frac{1}{2} t^{2}$

So $\lim _{t \rightarrow 0} \frac{\left(1+t^{2}\right)^{\frac{1}{3}}-1}{\cos t-1}=\lim _{t \rightarrow 0} \frac{\frac{1}{3} x^{2}}{-\frac{1}{2} x^{2}}=-\frac{2}{3}$.

Analysis: When calculate the limit by the equivalent infinitesimal, we must pay attention to than the independent variable tends to zero when using the equivalent infinitesimal.

\section{Calculation of limit by the continuity of a function}

Definition 2 the function $y=f(t)$ is defined in a neighborhood of the point $t_{0}$. If $\lim _{t \rightarrow t_{0}} f(t)=f\left(t_{0}\right)$, then Then function at this point is continued.

Theorem 4 every elementary function in the definition range is continued.

Example 5 Counting $\lim _{t \rightarrow 0} \frac{\sqrt{1+t^{2}}-1}{t}$

Analysis: The function

$$
f(t)=\frac{\sqrt{1+t^{2}}-1}{t}
$$

Is the elementary function, and $t=0$ is a point in the definition, so

$$
\lim _{t \rightarrow 0} \frac{\sqrt{1+t^{2}}-1}{t}=f(0)=0 \text {. }
$$

Calculation of limit by the continuity of a function, we must ensure that this function is defined at this point, can directly Substitute $\mathrm{x}$ to the function.

\section{Calculation of limit by the L'Hospital rule}

Theorem 5 When $t \rightarrow a$,

(1) The function $f(t)$ and $F(t)$ all tend to zero;

(2)In a deleted center neighborhood about the point $a, f^{\prime}(t)$ and $F^{\prime}(t)$ all exist, and $F^{\prime}(t) \neq 0$;

(3) $\lim _{t \rightarrow a} \frac{f(t)}{F(t)}=\lim _{t \rightarrow a} \frac{f^{\prime}(t)}{F^{\prime}(t)}$.

Example 6 Counting $\lim _{t \rightarrow 0} \frac{t-\sin t}{t^{3}}$

Count: When $t \rightarrow 0$ we can find that $t-\sin t \rightarrow 0$ and $t^{3} \rightarrow 0$ then

$$
\lim _{t \rightarrow 0} \frac{t-\sin t}{t^{3}}=\lim _{t \rightarrow 0} \frac{1-\cos t}{3 t^{2}}=\lim _{t \rightarrow 0} \frac{\sin t}{6 t}=\frac{1}{6} .
$$

In the example 6 , the numerator and denominator tend to zero, we can count the limit after counting the derivatives. If molecular and denominator all tend to zero in the same changing process, we can use the Hospital rule. 


\section{Calculation of limit by the two important limits}

The first important limit $\lim _{x \rightarrow 0} \frac{\sin x}{x}=1$

The second important limit $\lim _{x \rightarrow \infty}\left(1+\frac{1}{x}\right)^{x}=e\left(\right.$ or $\left.\lim _{x \rightarrow 0}(1+x)^{\frac{1}{x}}=e\right)$

The second important limit has three characteristics: When $x \rightarrow \infty$, we can see $\left(1+\frac{1}{x}\right)^{x}$ as $1^{\infty}$;

There must be " $1+$ "in the function" $\frac{1}{x}$ "and " $x$ "must be reciprocal in " $\left(1+\frac{1}{x}\right)^{x}$ "

Example 7 Counting $\lim _{x \rightarrow 0} \frac{\tan x}{x}=1$

Count: $\lim _{x \rightarrow 0} \frac{\tan x}{x}=\lim _{x \rightarrow 0}\left(\frac{\sin x}{x} \frac{1}{\cos x}\right)=\lim _{x \rightarrow 0}\left(\frac{\sin x}{x}\right) \lim _{x \rightarrow 0}\left(\frac{1}{\cos x}\right)=1$

Example 8 Counting $\lim _{x \rightarrow \infty}\left(1-\frac{1}{x}\right)^{x}$

Count: $\lim _{x \rightarrow \infty}\left(1-\frac{1}{x}\right)^{x}=\lim _{x \rightarrow \infty}\left[1+\left(-\frac{1}{x}\right)\right]^{x}=\lim _{x \rightarrow \infty}\left\{\left[1+\left(-\frac{1}{x}\right)\right]^{-x}\right\}^{-1}=e^{-1}$

\section{Calculation of limit by derivatives of the functions about the indefinite integral upper limit}

Theorem 6 If the function $f(x)$ is continuous on the section $[a, b]$,the function about the integral upper limit

has derivative, and its derivative is

$$
\phi(x)=\int_{a}^{x} f(t) d t
$$

$$
\phi^{\prime}(x)=\frac{d}{d x} \int_{a}^{x} f(t) d t=f(x)
$$

This theorem points to an important conclusion: the continuous functions $f(x)$ calculate the definite integral about the changing upper limit $x$.The result reductive itself $f(x)$.Associate the counting limit, we can use the L'Hospital rule to counting the limit. If the molecular or the denominator of the fractional appears this function of the indefinite integral upper limit, we can use the idea of the theorem 6 to count the derivation.

Example 9 Counting $\lim _{x \rightarrow 0} \frac{\int_{\cos x}^{1} e^{-t^{2}} d t}{x^{2}}$

Analysis: This is an uncertain formula clearly, we can count it by the L'Hospital rule.The fraction can be writtened to

$$
\int_{\cos x}^{1} e^{-t^{2}} d t=-\int_{1}^{\cos x} e^{-t^{2}} d t
$$

This integral's upper limit is $\cos x$, it is a function by $x$. It can be seen a compound function by the middle variable $u=\cos x$.According to the theorem 6, we can calculate:

So,

$$
\begin{gathered}
\frac{d}{d x} \int_{\cos x}^{1} e^{-t^{2}} d t=-\frac{d}{d x} \int_{1}^{\cos x} e^{-t^{2}} d t \\
=-\left.\frac{d}{d u} \int_{1}^{u} e^{-t^{2}} d t\right|_{u=\cos x} \square(\cos x)^{\prime} \\
=-e^{-\cos x^{2}} \square(-\sin x) \\
=\sin x e^{-\cos x^{2}}
\end{gathered}
$$

$$
\lim _{x \rightarrow 0} \frac{\int_{\cos x}^{1} e^{-t^{2}} d t}{x^{2}}=\lim _{x \rightarrow 0} \frac{\sin x e^{-\cos x^{2}}}{2 x}=\frac{1}{2 e} .
$$




\section{Calculation of limit by Taylor formula}

Taylor formula: If the function $f(x)$ has $(n+1)$ duplicates derivatives, then $\forall x \in(a, b)$,

$$
\begin{gathered}
f(x)=f\left(x_{0}\right)+f^{\prime}\left(x_{0}\right)\left(x-x_{0}\right)+\frac{f^{\prime \prime}\left(x_{0}\right)}{2 !}\left(x-x_{0}^{2}\right)+\cdots \cdots \\
+\frac{f^{(n)}\left(x_{0}\right)}{n !}\left(x-x_{0}\right)^{n}+R_{n}(x)
\end{gathered}
$$

$R_{n}(x)=\frac{f^{(n+1)}(\xi)}{(n+1) !}\left(x-x_{0}\right)^{n+1}$, this $\xi$ is a value between $x$ and $x_{0}$.

Example 10 Counting the limit by the McLaughlin formula who has the penano remainder: $\lim _{x \rightarrow 0} \frac{\sin (x)-x \cos x}{\sin ^{3} x}$

Count: As the denominator of the fractional $\sin ^{3} x \sim x^{3}(x \rightarrow 0)$, we can only use the McLaughlin formula

$$
\sin x=x-\frac{x^{3}}{3 !}+o\left(x^{3}\right), x \cos x=x-\frac{x^{3}}{2 !}+o\left(x^{3}\right)
$$

Substitute:

$$
\sin x-x \cos x=x-\frac{x^{3}}{3 !}+o\left(x^{3}\right)-x+\frac{x^{3}}{2 !}-o\left(x^{3}\right)=\frac{x^{3}}{3}+o\left(x^{3}\right)
$$

In the above formula, we can think the two $o\left(x^{3}\right)$ as one $o\left(x^{3}\right)$.

\section{The conclusion}

The above methods are just some main method for counting limit, some specific problems have specific analysis. For example, some limits need to simplify and then substitute; some limits need to change element; some limits even need rational the molecular denominator etc. For the limit of piecewise function at the interrupted point. We need count the limit on the left and right side of this point then do the judgment. If you want to be familiar with t the method of counting the limit, you need do the various types of exercises, then lay the good foundation on the Calculus.

\section{Acknowledgements}

This work is supported by National Nature Science Foundation of Shandong education province planning project on 2013(2013GG210).

This work is supported by National Nature Science Foundation of the informational and electrical engineering professional platform’s course on Zaozhuang College (2014C13).

\section{References}

[1] Department of Mathematics in tong ji University. Higher Mathematics (Reference of Higher Education, China 2007).

[2] Jianfu Wang. The Synchronous Tutorship and The Exercise's Question on Higher Mathematics(Reference of Chinese Mining and Technology University, China 2008).

[3] Wenlin Li. An introduction to the mathematics history (Reference of Higher Education, China 2011). 